Catastrophizing Scale (PCS, 0-52 scale) at the baseline examination in 20162017. Bilateral interphalangeal, metacarpophalangeal, first carpometacarpal and the scaphotrapeziotrapezoidal joint were scored for radiographic OA according to the Kellgren-Lawrence (KL) index (sum score: 0-132 scale). Using linear regression analyses, we analysed whether KL sum score, HADS sum score and PCS sum score (independent variables) were associated with AUSCAN pain (dependent variable). Separate models were applied for each independent variable with adjustment for age, sex and body mass index (BMI). Thereafter, all independent variables were included in the same model. Analyses were repeated using NRS hand pain as the dependent variable. Interactions between $\mathrm{KL}$ sum score and HADS/PCS were explored.

Results: Patients reported wide range of pain severity with mean (SD) AUSCAN pain of 8.2 (4.0) and mean (SD) NRS hand pain of 3.8 (2.3). Their radiographic severity ranged from minimal to severe with a median (IQR) KL sum score of 28 (15-44). Most patients reported low levels of anxiety, depression and pain catastrophizing with median (IQR) HADS sum score of 6 (3-10) and PCS sum score of $9(5-15)$.

The HADS and PCS sum scores were associated with hand pain, both when analysed separately and in the same model together with $\mathrm{KL}$ sum score (table 1). Increasing radiographic severity was not associated with hand pain in the age, sex and BMI-adjusted models. When including HADS and PCS in the models, the associations between radiographic severity and pain became stronger for both pain outcomes and statistically significant for AUSCAN pain (table 1).

We found interactions between KL sum score and HADS. In the 61 persons with HADS depression and anxiety subscale scores below 8 , the $\mathrm{KL}$ sum score was significantly associated with AUSCAN pain $(B=0.03,95 \% \mathrm{Cl}: 0.003$ to 0.06 , $\mathrm{p}=0.03$ ) and NRS pain ( $\mathrm{B}=0.02,95 \% \mathrm{Cl}: 0.002$ to $0.03, \mathrm{p}=0.03$ ) (adjusted for age, sex, BMI and PCS), whereas no associations between radiographic severity and pain was found in persons with HAD depression and/or anxiety subscale scores of 8 or more.

Abstract OP0054 - Table 1

\begin{tabular}{|l|l|l|l|l|}
\hline & \multicolumn{2}{|l|}{ AUSCAN pain $(\mathrm{B}, 95 \% \mathrm{Cl})$} & \multicolumn{2}{l|}{ NRS hand pain $(\mathrm{B}, 95 \% \mathrm{Cl})$} \\
\hline & Model $1^{*}$ & Model $2 * *$ & Model $1^{*}$ & Model $2^{* *}$ \\
\hline KL sum score & $0.01(-0.01,0.04) ;$ & $0.03(0.001,0.05)$, & $0.005(-0.01,0.02) ;$ & $0.01(-0.002,0.03) ;$ \\
& $p=0.35$ & $p=0.04$ & $p=0.52$ & $p=0.09$ \\
\hline HADS sum & $0.19(0.11,0.26) ;$ & $0.12(0.04,0.20) ;$ & $0.11(0.07,0.15) ;$ & $0.08(0.03,0.12) ;$ \\
score & $p<0.001$ & $p=0.01$ & $p<0.001$ & $p=0.001$ \\
\hline PCS sum score & $0.17(0.12,0.23) ;$ & $0.14(0.08,0.20) ;$ & $0.10(0.07,0.13) ;$ & $0.07(0.04,0.11) ;$ \\
& $p<0.001$ & $p<0.001$ & $p<0.001$ & $p<0.001$ \\
\hline
\end{tabular}

HADS and PCS isclud KL, HADS and PCS, respectively, with adj. for age, sex

Conclusions: Depression, anxiety and pain catastrophizing were associated with pain in hand OA, emphasising that pain in hand OA should be treated in a biopsychosocial framework. Importantly, radiographic severity was associated with pain only in persons with no or low levels of depression and anxiety.

Disclosure of Interest: None declared

DOI: 10.1136/annrheumdis-2018-eular.1939

\section{OP0055 A NOVEL METHOD FOR ASSESSING PROXIMAL TIBIOFIBULAR JOINT ON MR IMAGES IN PATIENTS WITH KNEE OSTEOARTHRITIS}

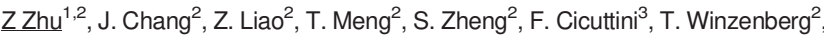
A. Wluka ${ }^{3}$, D. Jiang ${ }^{4}$, W. Han ${ }^{2}$, C. Ding ${ }^{1,2} .{ }^{1}$ Clinical Research Center, Zhujiang Hospital, Guangzhou, China; ${ }^{2}$ Menzies Institute for Medical Research, University of Tasmania, Hobart, ${ }^{3}$ Department of Epidemiology and Preventive Medicine, Monash University, Melbourne; ${ }^{4}$ School of Engineering and ICT, University of Tasmania, Hobart, Australia

Background: Proximal tibiofibular joint (ProxTibFibJ) is a synovial sliding joint that has been estimated to transmit one-sixth of the leg's static load. One study has reported that proximal fibular osteotomy could significantly improve the clinical outcomes in patients with medial compartment OA. However, no study has delineated the measurement of ProxTibFibJ morphological parameters (ProxTibFibJ contacting area, load-bearing area, lateral stress-bolstering area and posterior stress-bolstering area) on magnetic resonance imaging (MRI) and investigated their correlations with knee OA structural abnormalities.

Objectives: To validate a pragmatic method to measure the morphological parameters of the ProxTibFibJ and to describe their associations with knee structural abnormalities in patients with knee osteoarthritis $(\mathrm{OA})$.

Methods: A total of 408 participants with knee OA were selected. The morpholog ical status of ProxTibFibJ were measured on coronal and sagittal magnetic resonance images (MRI). We calculated the contacting area of ProxTibFibJ (S), and its projection areas onto the horizontal (load-bearing area, $\mathrm{S} \tau$ ), sagittal (lateral stress-bolstering area, $S \varphi$ ) and coronal plane (posterior stress-bolstering area, $\mathrm{S} v)$, respectively. Knee structural abnormalities including cartilage defects, bone marrow lesions (BMLs) and cartilage volume were evaluated. Clinical construct validity was examined through describing the associations between the morphological parameters of ProxTibFibJ and knee structural abnormalities. The reliabilities were examined by calculating the intra- and inter-observer correlation coefficients.

Results: The average ProxTibFibJ fibular contacting area was $2.4 \pm 0.7 \mathrm{~cm}^{2}$. The intra- and inter-observer correlation coefficients for all measures were excellent (all $\geq 0.90$ ). In cross-sectional analyses, the ProxTibFibJ morphological parameters $(\mathrm{S}, \mathrm{S} \tau, \mathrm{S} v$ and $\mathrm{S} \varphi)$ were significantly associated with radiographic medial JSN (OR 1.72 for $S ; 2.20$ for $\mathrm{S} \tau ; 1.65$ for $\mathrm{S} v$ ), radiographic medial osteophyte (OR 0.51 for $\mathrm{S} \varphi$ ) and $\mathrm{MRI}$-assessed knee joint structural abnormalities including cartilage volume ( $\beta-0.07$ for $\mathrm{S} ;-0.09$ for $\mathrm{S} \tau$ ), cartilage defects (OR 1.63 for $\mathrm{S} ; 1.95$ for $\mathrm{S} \tau$ ) and BMLs (OR 1.54 for $\mathrm{S} ; 1.74$ for $\mathrm{S} \tau$ ) at medial tibiofemoral compartment. In longitudinal analyses, $S(R R, 1.45)$ and $S \tau(R R, 1.55)$ of ProxTibFibJ were significantly and positively associated with an increase in medial tibial cartilage defects over 2 years, after adjustment for age, gender, height, weight, ROA, tibia plateau bone area and intervention. $\mathrm{S} \tau(\beta,-0.07), \mathrm{S} v(\beta,-0.07)$ and $\mathrm{S}(\beta,-0.06)$ of ProxTibFibJ were significantly and negatively associated with change in medial tibial cartilage volume, after adjusted for above covariates. S $\tau(R R, 1.55)$ of ProxTibFibJ was positively associated with an increase in medial tibial BML, and S (RR, 0.35) was negatively associated with an increase in medial femoral BMLs.
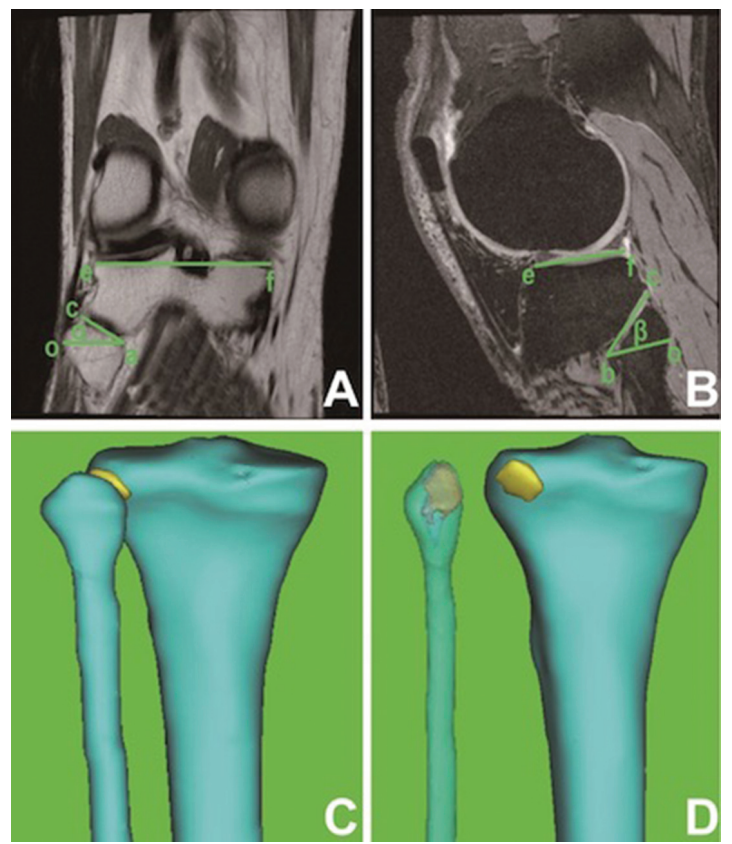

Conclusions: This novel method to assess the morphological parameters of ProxTibFibJ using MRI is reproducible, and has clinical construct validity. The longitudinal associations with osteoarthritic changes suggest that higher load-bearing area of ProxTibFibJ is a potential risk factor for medial compartment OA.

Disclosure of Interest: None declared

DOI: 10.1136/annrheumdis-2018-eular.1685

\section{OP0056 MOLECULAR AND STRUCTURAL BIOMARKERS OF INFLAMMATION AT 2 YEARS AFTER ACUTE ACL INJURY DO NOT PREDICT STRUCTURAL KNEE OSTEOARTHRITIS AT 5 YEARS}

F Roemer ${ }^{1,2,3}$, M. Englund $^{4}$, A. Turkiewicz ${ }^{4}$, A. Struglics ${ }^{1}$, A. Guermazi ${ }^{2}$, L. S. Lohmander ${ }^{1}$, S. Larsson ${ }^{1}$, R. Frobell ${ }^{1}{ }^{1}$ Orthopaedics, Department of Clinical Sciences Lund, Lund University, Lund, Sweden; ${ }^{2}$ Radiology, Boston University School of Medicine, Boston, USA; ${ }^{3}$ Radiology, University of Erlangen-Nuremberg, Erlangen, Germany, ${ }^{4}$ Clinical Epidemiology Unit, Orthopaedics, Department of Clinical Sciences Lund, Lund University, Lund, Sweden

Background: Trauma-induced cytokine response and local inflammation after knee injury may be important in the development of posttraumatic osteoarthritis $(\mathrm{OA})$. It has been reported that synovial fluid levels of inflammatory markers remain increased up to 5 years after anterior cruciate ligament $(A C L)$ injury, indicative of extended local inflammation in the injured joint. ${ }^{1}$ Inflammation potentially represents a valid target for treatment in the early and subacute phase after joint trauma in order to prevent or delay onset of post-traumatic knee OA. 